\title{
The effect of COVID-19 disease on sperm parameters and female fertility
}

\section{ARTICLE INFO}

Article Type

Review of article

Authors

Javad Amini Mahabadi ${ }^{1,2^{*}}$ (iD), PhD

${ }^{1} \mathrm{PhD}$ of reproductive biology, Gametogenesis Research Center, Kashan University of Medical

Sciences, Kashan, Iran.

${ }^{2}$ Sarem Fertility \& Infertility

Research Center (SAFIR) \&

Sarem Cell Research Center

(SCRC), Sarem Women's

Hospital, Iran University of

Medical Sciences (IUMS),

Tehran, Iran.

\section{*Corresponding Author}

Address: Gametogenesis Research Center, Kashan University of Medical Sciences, Kashan, Iran and

Sarem Fertility \& Infertility

Research Center (SAFIR), Iran University of Medical Sciences,

Tehran, Iran.

Phone: +989132770917

j.mahabadi64@gmail.com

\section{ABSTRACT}

Aims: The COVID-19 pandemic is an extraordinary global situation, and all countries have adopted their own strategies to diminish and eliminate the spread of the virus. Scientifc societies, such as the European Society for Human Reproduction and Embryology and American Society for Reproductive Medicine, have provided recommendations and guidance to overcome on infection in patients who undergo IVF treatments. Although there is as yet no evidence that the virus causing COVID-19 might have negative effects on IVF outcomes, but in order to support healthcare systems fertility treatments have been postponed approximately. Methods: In this review paper, included studies were investigated in Persian and English databases, such as SID, PubMed, Google scholar, Science direct, Embase, and Scopus, as well as clinical protocols and reports of reputable health organizations during 2000 to 2021 that related with different types of coronaviruses. The search terms were "Pregnancy", "Coronavirus", "novel Coronavirus", "Covid-19", "female reproductive system", and "IVF" that collectively, 21 studies were evaluated in this study.

Conclusion: Due to the global prevalence of this virus, protocols, health care and preventive measures should be taught at the community level in the relationship wiht fertility and infertility centers, and monitoring systems should be considered to this disease, so that these persons can be specifically monitored and followed up during pregnancy.

Keywords: Coronaviruses; Covid-19; Female reproductive system; IVF.

\section{Article History}

Received: January 19, 2021

Accepted: February 03, 2021

e Published: August 11, 2021

Copyright(C) 2021, ASP Ins. This open-access article is published under the terms of the Creative Commons Attribution-Noncommercial 4.0 International License which permits Share (copy and distribute the material in any medium or format) and Adapt (remix, transform, and build 
كليد وازمها: كروناويروس ها؛ كوويد-9 1؛ سيستم توليدمثلى زنان؛ IVF.

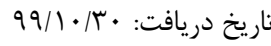

تاريخ پذيرش:

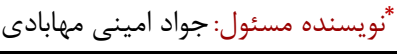

مقدمه

در اواخر سال 9 أ r، ويروس كروناى جديد، SARS-CoV-2، در جين

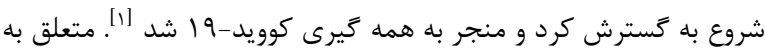

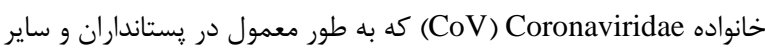
حيوانات وجود دارند، ويروس هاى كرونا باعث سندرم حاد تنفسى حاد

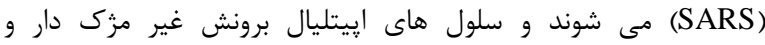

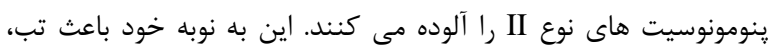

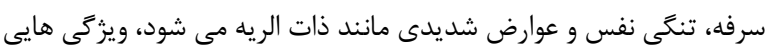

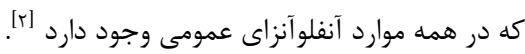

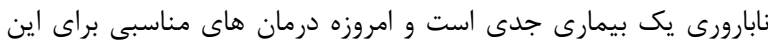

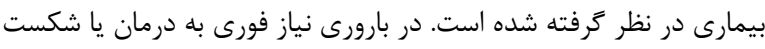

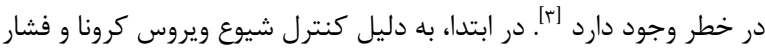

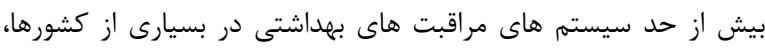

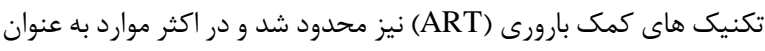

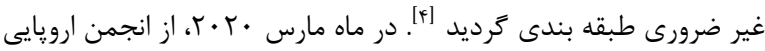

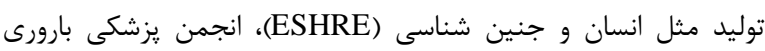

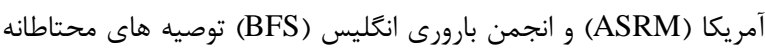

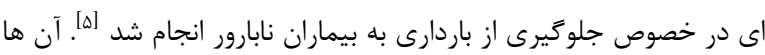

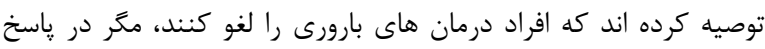

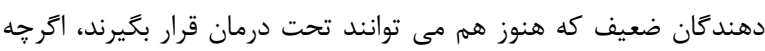

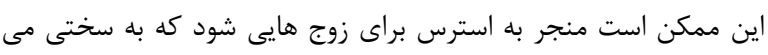

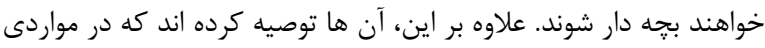

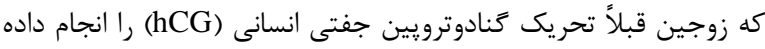

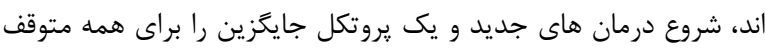

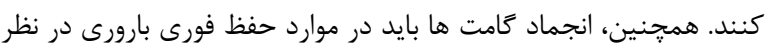

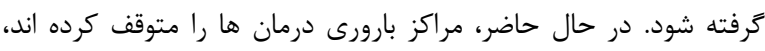

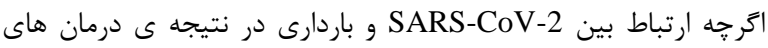

ART

اطلاعات كمى در مورد احتمال آلودگى اسيرم، تخمك يا جنين، رِتانسيل

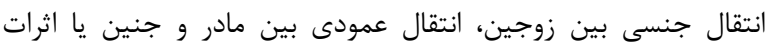

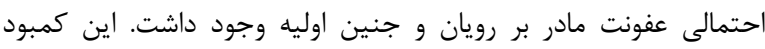

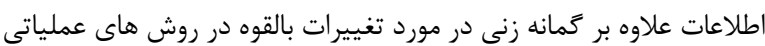

تاثير بيمارى كوويد-19 بر سيستم توليدمثلى و بارورى زنان

جكيده

اهداف: همه كيرى بيمارى كوويد-9 ايكى وضعيت جهانى فوق العاده است و همه كشورها استراتزى هاى خاص خود را براى كاهش و از بين بردن

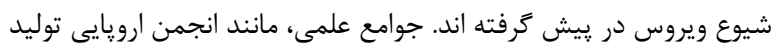

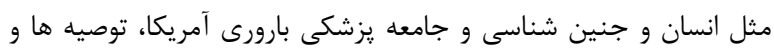

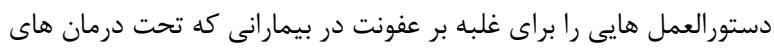

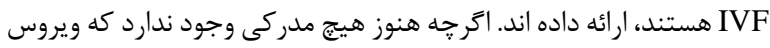

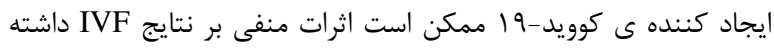

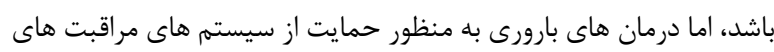
بهداشتى تا حدودى به تعويق افتاده است.

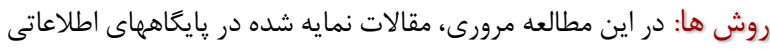

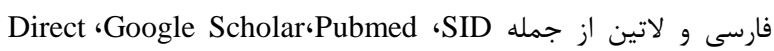
Scopus ،Embase ،Science

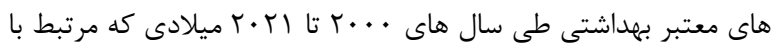

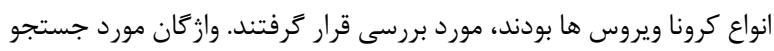

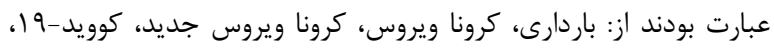

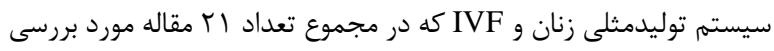
قرار گرفت. نتيجه گيرى: با توجه به شيوع جهانى اين ويروس، يروتكلها، مراقبتهاى

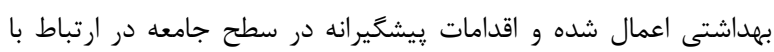

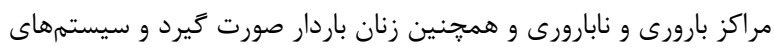

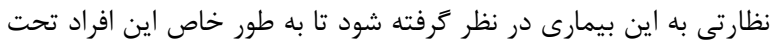
نظر و يُيخيرى هاى درمانى دوران باردارى قرار گيرند. 


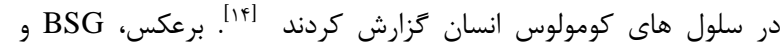

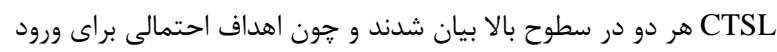

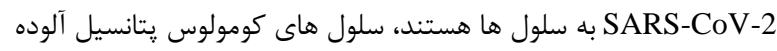

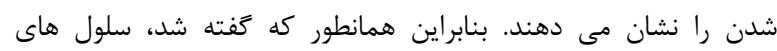

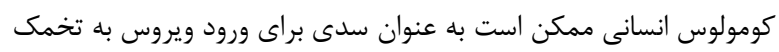

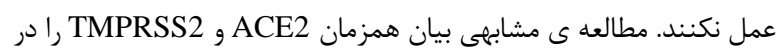

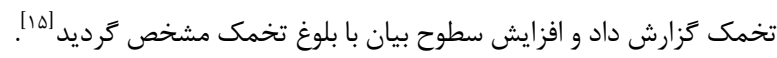

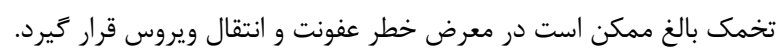

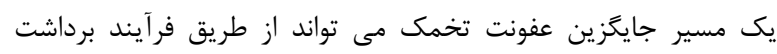

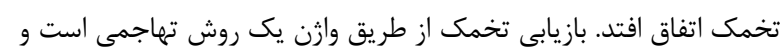

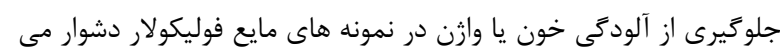

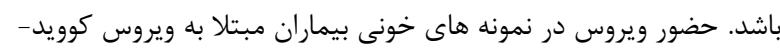

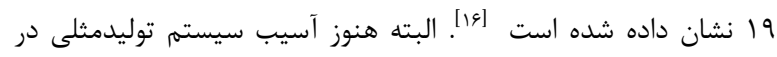

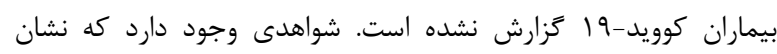

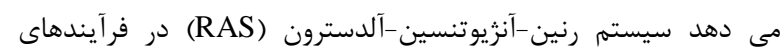

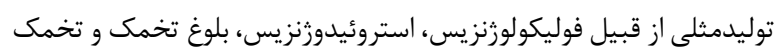

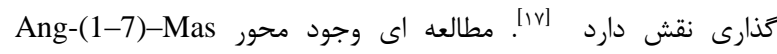

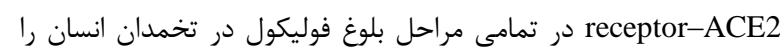

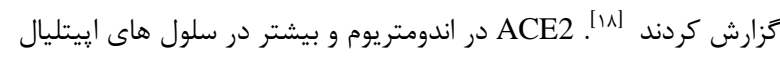

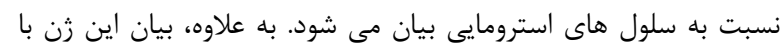

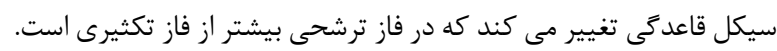

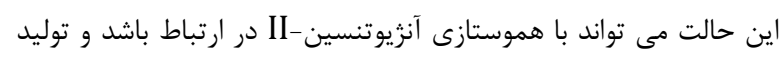

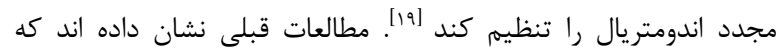
ACE2 مى شود [ᄉ/و.r.

\section{ارتباط بين كوويد-19 و IVF}

انجام IVF در حضور ميكروار كانيسم هاى عفونى براى مراكز بارورى مبحث

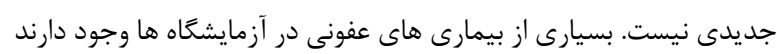

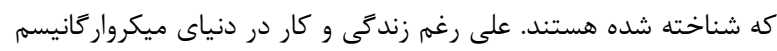

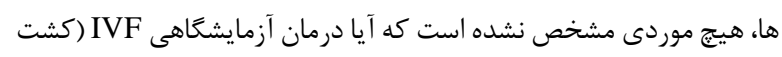

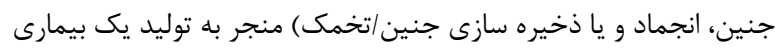

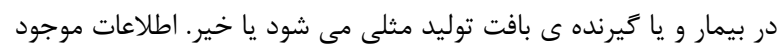

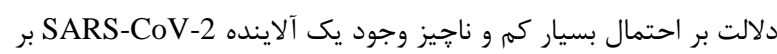

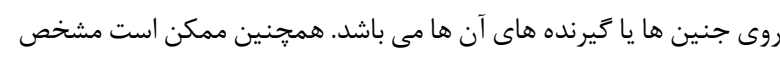

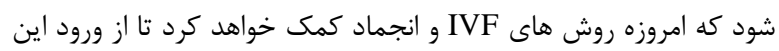

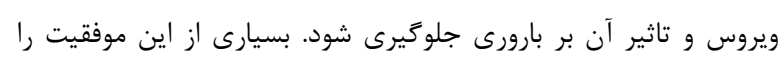

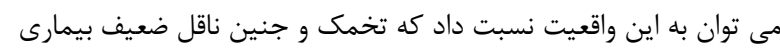

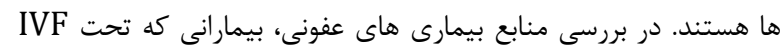

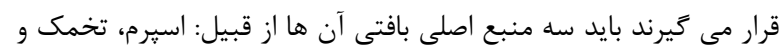

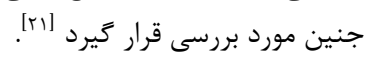

استاندارد (SOPs) در آزمايشعاه IVF در طى همه كيرى، باعث نكرانى

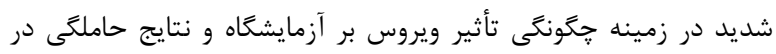

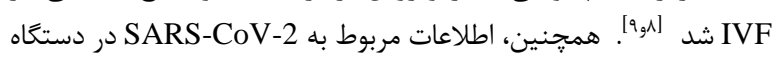

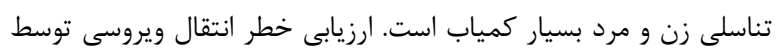

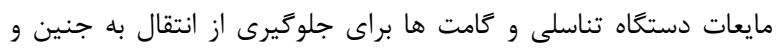

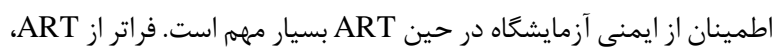

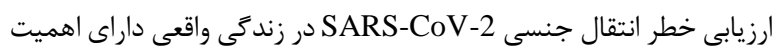

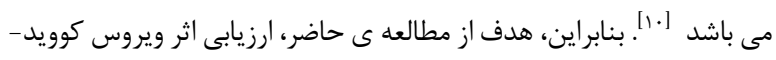
19 بر تخمك و بارورى زنان است.

ارتباط بين كوويد-19 و توليدمثل انسان

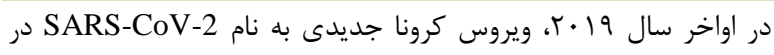

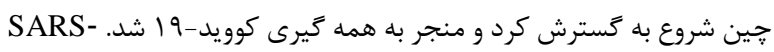
CoV-2

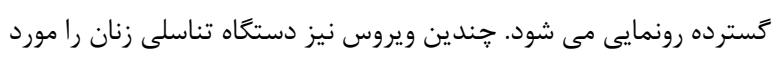

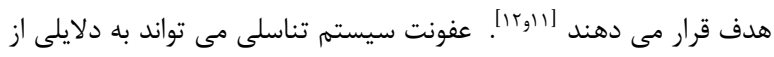

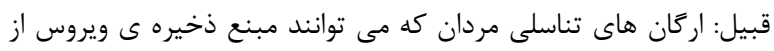

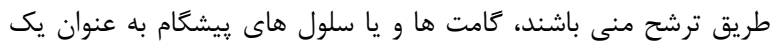

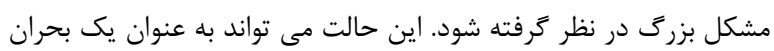

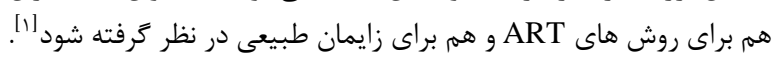

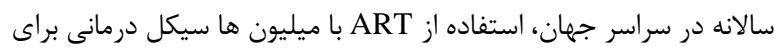

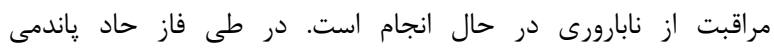

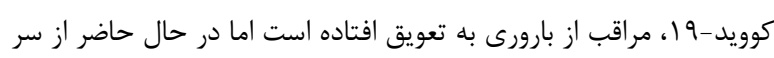

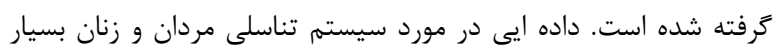

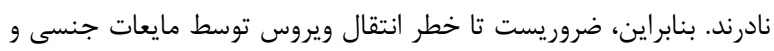

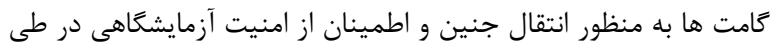

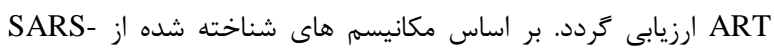

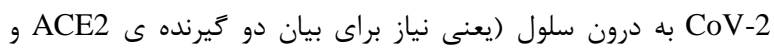
TMPRSS2

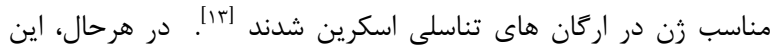

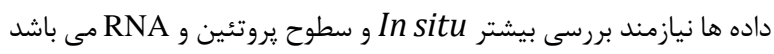

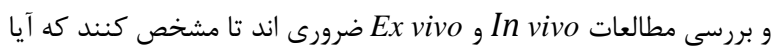
SARS-CoV-2

\section{ارتباط بين كوويد-19 و تخمك}

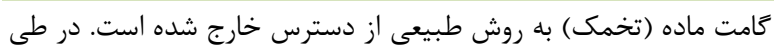

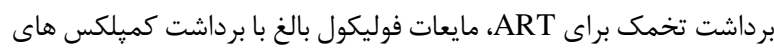

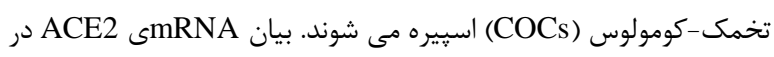

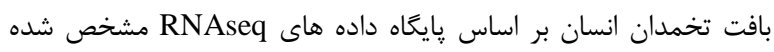

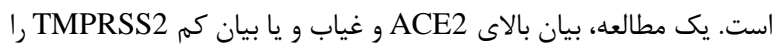


تميز كردن بايد با احتياط انجام شود، ماند ضد عفونى كردن سطوح مشترك (به عنوان مثال، دستخيره هاى در، صفحه كليد، سطوح وغيره)

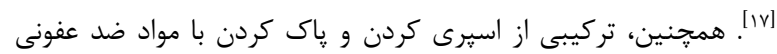

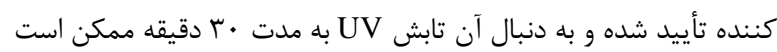

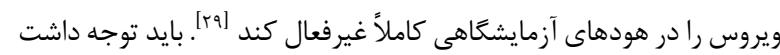

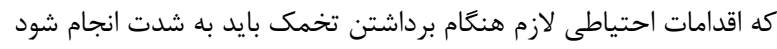

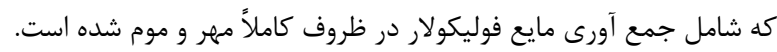

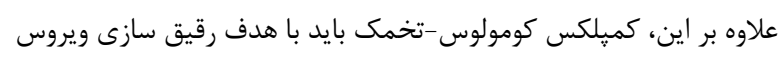

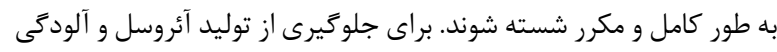

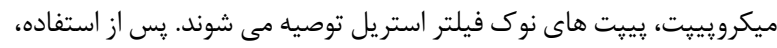

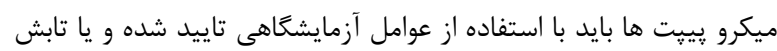

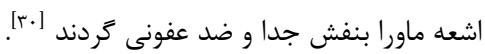

\section{نتيجه تيرى}

شيوع بيمارى كوويد-19 و تعداد موارد مرى و مير ناشى از آن در سراسر

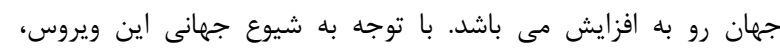

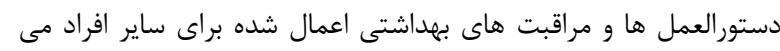

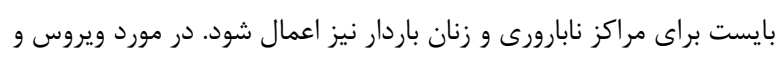

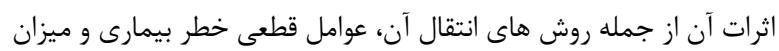

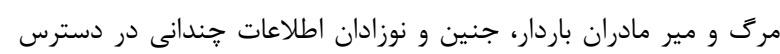

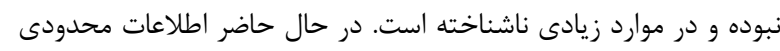

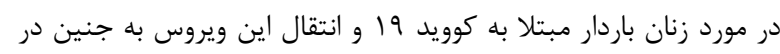

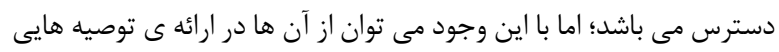

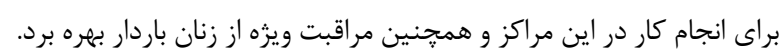

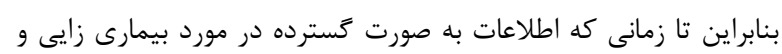

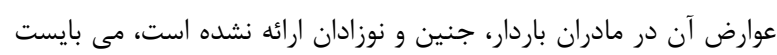

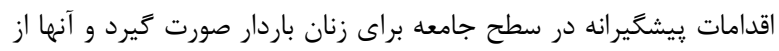

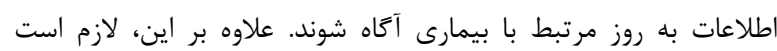

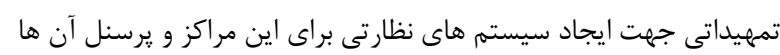

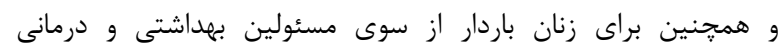
كشورصورت كيرد.

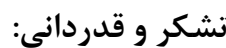

بدين وسيله از بيمارستان و مركز تحقيقات بارورى و نابارورى صارم تقدير و تشكر به عمل مى آيد.

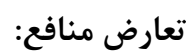

در اين مطالعه تعارض منافع وجود نداشت.
پاسخ اين كه آيا جنين ها مى توانند توسط ويروس SARS-CoV-2 آلوده

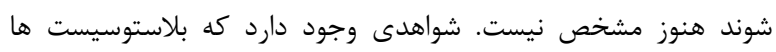

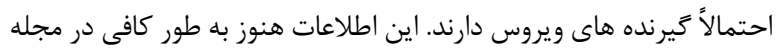

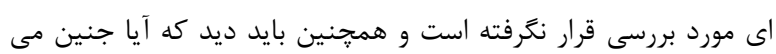

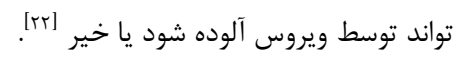
محققان خطرات انتقال بيمارى از طريق جنين را مورد بر رسى قران قرار داده اند.

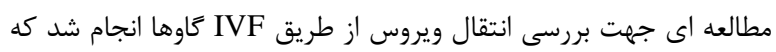
توسط فلاويروس ها عفونى شده بودند. علارغم يافته هاى ويروس در در مايع إنقائ

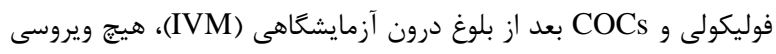

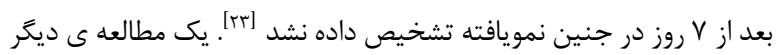

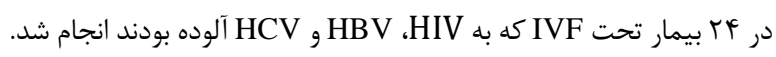

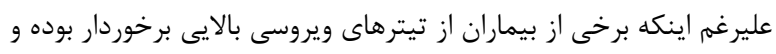

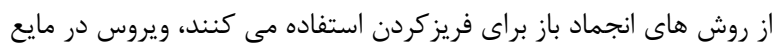

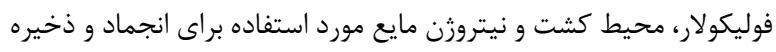

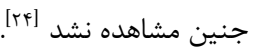

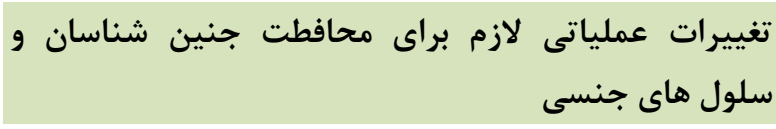
از آنجا كه ويروس هاى كرونا در خون جيسى بيماران آلوده و با توجه به وجود

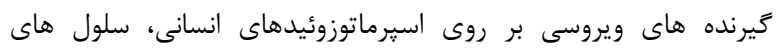
سرتولى، سلول هاى لايديخ و تخمك ها تشخيص داده شده اند، هوشيارى

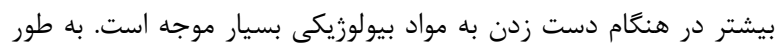

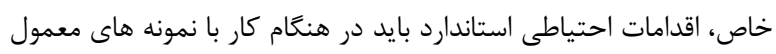

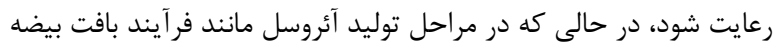

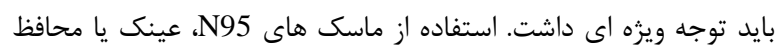

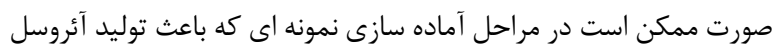

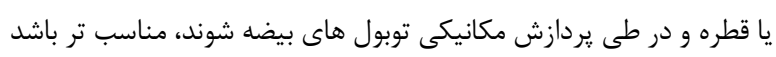

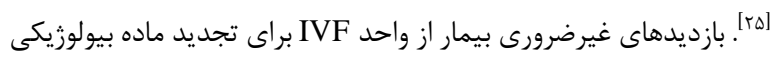

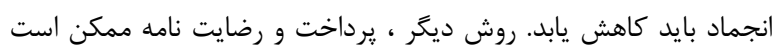

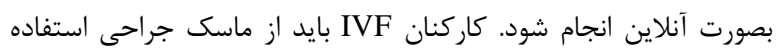

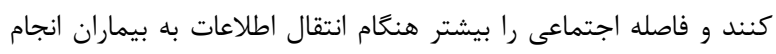

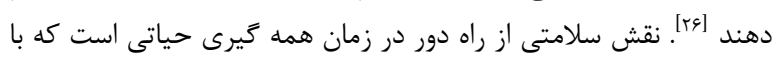

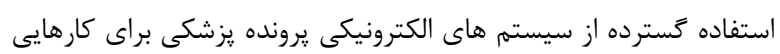

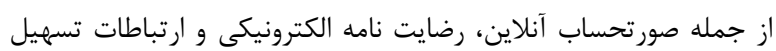

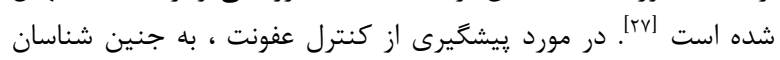

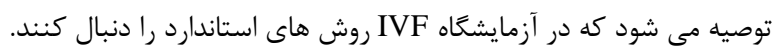

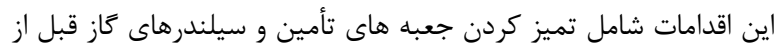

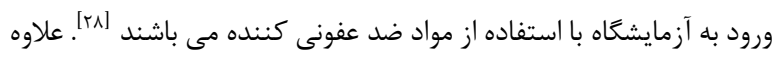

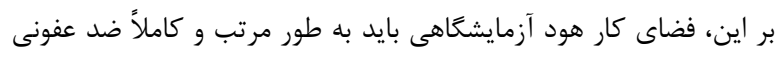

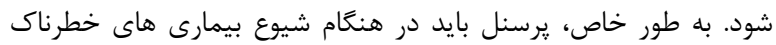

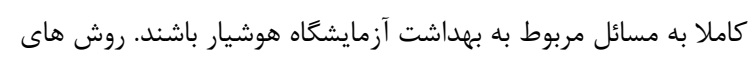


12. Bujan L, Mansuy J-M, Hamdi S, Pasquier C, Joguet G. 1 year after acute Zika virus infection in men. Lancet Infect Dis. 2020;20(1):25-6.

13. Holtmann N, Edimiris P, Andree M, Doehmen C, Baston-Buest D, Adams O, et al. Assessment of SARS-CoV-2 in human semen-a cohort study. Fertil Steril. 2020;114(2):233-8.

14. Stanley KE, Thomas E, Leaver M, Wells D. Coronavirus disease (COVID-19) and fertility: viral host entry protein expression in male and female reproductive tissues. Fertil Steril. 2020;

15. Yang M, Chen S, Huang B, Zhong J-M, Su H, Chen Y-J, et al. Pathological findings in the testes of COVID-19 patients: clinical implications. Eur Urol Focus. 2020;6(5):1124-9.

16. Scorzolini L, Corpolongo A, Castilletti C, Lalle E, Mariano A, Nicastri E. Comment on the Potential Risks of Sexual and Vertical Transmission of COVID-19. Clin Infect Dis. 2020;71(16):2298.

17. Li R, Yin T, Fang F, Li Q, Chen J, Wang Y, et al. Potential risks of SARS-Cov-2 infection on reproductive health. Reprod Biomed Online. 2020;

18. Pereira VM, Reis FM, Santos RAS, Cassali GD, Santos SHS, Honorato-Sampaio K, et al. Gonadotropin stimulation increases the expression of angiotensin-(1-7) and MAS receptor in the rat ovary. Reprod Sci. 2009;16(12):1165-74.

19. Vaz-Silva J, Carneiro MM, Ferreira MC, Pinheiro SVB, Silva DA, Silva AL, et al. The vasoactive peptide angiotensin-(1-7), its receptor Mas and the angiotensin-converting enzyme type 2 are expressed in the human endometrium. Reprod Sci. 2009;16(3):247-56.

20. Barreta MH, Gasperin BG, Ferreira R, Rovani M, Pereira GR, Bohrer RC, et al. The components of the angiotensin-(1-7) system are differentially expressed during follicular wave in cattle. J Renin-Angiotensin-Aldosterone Syst. 2015;16(2):275-83.

21. Pomeroy KO, Schiewe MC. Cryopreservation and IVF in the time of Covid-19: what is the best good tissue practice (GTP)? J Assist Reprod Genet. 2020;37(10):2393-8.

22. Colaco S, Chhabria K, Singh N, Bhide A, Singh D, Singh A, et al. Expression of SARS-CoV-2 receptor ACE2 and the spike protein processing enzymes in developing human embryos. arXiv Prepr arXiv200404935. 2020;

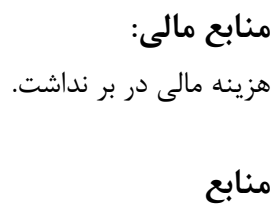

1. Entezami F, Samama M, Dejucq-Rainsford N, Bujan L. SARS-CoV-2 and human reproduction: An open question. EClinicalMedicine. 2020;25.

2. Ashour HM, Elkhatib WF, Rahman M, Elshabrawy HA. Insights into the recent 2019 novel coronavirus (SARS-CoV-2) in light of past human coronavirus outbreaks. Pathogens. 2020;9(3):186.

3. Zegers-Hochschild F, Adamson GD, Dyer S, Racowsky C, De Mouzon J, Sokol R, et al. The international glossary on infertility and fertility care, 2017. Hum Reprod. 2017;32(9):1786-801.

4. Rajput SK, Logsdon DM, Kile B, Engelhorn HJ, Goheen B, Khan S, et al. Human eggs, zygotes, and embryos express the receptor ACE2 and protease TMPRSS2 protein necessary for SARS-CoV-2 infection. F\&S Sci. 2020;

5. Robertson I, Kermack AJ, Cheong Y. The impact of Covid-19 on infertility services and future directions. Reprod Fertil. 2020;1(1):C3-7.

6. La Marca A, Niederberger C, Pellicer A, Nelson SM. COVID-19: lessons from the Italian reproductive medical experience. Fertil Steril. 2020;113(5):920.

7. Rodriguez-Wallberg KA, Wikander I. A global recommendation for restrictive provision of fertility treatments during the COVID-19 pandemic. Acta Obstet Gynecol Scand. 2020;99(5):569.

8. Anifandis G, Messini CI, Daponte A, Messinis IE. COVID-19 and fertility: a virtual reality. Reprod Biomed Online. 2020;41(2):157-9.

9. Arav A. A recommendation for IVF lab practice in light of the current COVID-19 pandemic. J Assist Reprod Genet. 2020;37:1543.

10. Li D, Jin M, Bao P, Zhao W, Zhang S. Clinical characteristics and results of semen tests among men with coronavirus disease 2019. JAMA Netw open. 2020;3(5):e208292-e208292.

11. Le Tortorec A, Matusali G, Mahé D, Aubry F, Mazaud-Guittot S, Houzet L, et al. From ancient to emerging infections: the odyssey of viruses in the male genital tract. Physiol Rev. 2020;100(3):1349-414. 
23. Bielanski A, Dubuc C. In vitro fertilization of ova from cows experimentally infected with a noncytopathic strain of bovine viral diarrhea virus. Anim Reprod Sci. 1995;38(3):215-21.

24. Cobo A, Bellver J, de los Santos MJ, Remohí J. Viral screening of spent culture media and liquid nitrogen samples of oocytes and embryos from hepatitis B, hepatitis $\mathrm{C}$, and human immunodeficiency virus chronically infected women undergoing in vitro fertilization cycles. Fertil Steril. 2012;97(1):74-8.

25. Van der Sande M, Teunis P, Sabel R. Professional and home-made face masks reduce exposure to respiratory infections among the general population. PLoS One. 2008;3(7):e2618.

26. Choucair F, Younis N, Hourani A. IVF laboratory COVID-19 pandemic response plan: a roadmap. Middle East Fertil Soc J. 2020;25(1):1-7.

27. Hernández C, Valdera CJ, Cordero J, López E, Plaza J, Albi M. Impact of telemedicine on assisted reproduction treatment in the public health system. J Healthc Qual Res. 2020;35(1):27-34.

28. Technology S for AR. Laboratory guidance for commencing or continuing ART operations during the ongoing COVID-19 pandemic. 2020.

29. Kariwa H, Fujii N, Takashima I. Inactivation of SARS coronavirus by means of povidone-iodine, physical conditions and chemical reagents. Dermatology. 2006;212(Suppl. 1):119-23.

30. Group EC-19 W, Gianaroli L, Ata B, Lundin K, Rautakallio-Hokkanen S, Tapanainen JS, et al. The calm after the storm: re-starting ART treatments safely in the wake of the COVID-19 pandemic. Hum Reprod. 2021;36(2):275-82. 